\title{
Rinitis atrófica
}

\section{Atrophic rhinitis}

\author{
Natalia Cabrera S1, P Samanta Ruz², Constanza J. Valdés².
}

\begin{abstract}
RESUMEN
La rinitis atrófica es una enfermedad crónica y progresiva de etiología desconocida. Se caracteriza por atrofia de la mucosa nasal y hueso subyacente, dilatación anormal de las cavidades nasales, obstrucción nasal paradójica, y formación de secreciones viscosas y costras secas; produciendo fetidez. Sus manifestaciones clínicas más frecuentes son obstrucción nasal, secreción purulenta, costras nasales y mal olor nasal.

Se ha separado en dos entidades: primaria y secundaria. El tratamiento es principalmente conservador, y se han propuesto diversas terapias farmacológicas y quirúrgicas.

La rinitis atrófica unilateral es una condición infrecuente, con escasos reportes en la literatura científica. Se puede asociar a la desviación septal, por lo que su corrección quirúrgica es una alternativa terapéutica disponible.
\end{abstract}

Palabras clave: Rinitis atrófica, ocena, turbinectomía, desviación septal.

\begin{abstract}
Atrophic rhinitis is a chronic disease of unknown etiology. This condition is characterized by progressive nasal mucosal and underlyng bone atrophy, abnormal widening of the nasal cavities, paradoxical nasal congestion and formation of viscid secretions and dried crusts, leading to a characteristic fetor (ozaena). The main clinical manifestations include nasal obstruction, purulent discharge, daily nasal crusting, nasal dryness and foul smell.

It has been divided into two separate entities; primary and secondary. Treatment is mostly conservative, although pharmacological and surgical therapies have been proposed.

Unilateral atrophic rhinitis is an uncommon condition, with few reports in the scientific literature. It is associated with septum deviation, so surgical correction is one of the therapeutic options available.
\end{abstract}

Key words: Atrophic rhinitis, Ozaena, Turbinectomy, Septum deviation.

\footnotetext{
${ }^{1}$ Interna de Medicina, Universidad de Chile.

${ }^{2}$ Médico Otorrinolaringólogo, Departamento de Otorrinolaringología Universidad de Chile. Hospital del Salvador.
} 


\section{INTRODUCCIÓN}

La Rinitis Atrófica (RA) es una enfermedad crónica de la mucosa de la cavidad nasal de etiología desconocida'. Esta condición es progresiva y se caracteriza por la atrofia de la mucosa nasal y del hueso subyacente de los cornetes, con dilatación anormal de las cavidades nasales, congestión nasal paradójica, y formación de secreciones viscosas y costras secas que conducen a una fetidez característica, llamada comúnmente ocena ${ }^{1,2}$.

La RA se ha separado en dos entidades. Por un lado, la rinitis atrófica primaria que tiene un inicio espontáneo, progresión lenta y etiología no especificada; y por otro, la rinitis atrófica secundaria que se desarrolla después de cirugías reductoras nasales (por ej. turbinectomía radical), traumatismos nasales o sinusales, radioterapia, o rinosinusitis crónica en asociación con enfermedades granulomatosas crónicas de la nariz ${ }^{2,3}$. La incidencia de la RA primaria ha disminuido notablemente en el último siglo, probablemente en relación con el aumento del uso de antibióticos para las infecciones crónicas nasales; pero sigue siendo una condición común en países como India, China, Egipto, entre otros ${ }^{1,2}$. Siendo la incidencia general es de $0,3 \%-7,8 \%$ en áreas afectadas ${ }^{4}$.

\section{ETIOLOGÍA}

RA primaria. La etiología es desconocida ${ }^{1,2,4,5}$. El diagnóstico es fundamentalmente clínico y de exclusión de las otras condiciones que pueden producir RA secundaria. Se han descrito diversos factores etiológicos posiblemente asociados con el desarrollo de RA primaria ${ }^{2,4}$ (Tabla 1). La infección se ha considerado como un factor principal, pero a menudo es difícil determinar si el microorganismo identificado es el agente causal de la destrucción de los tejidos 0 si los microorganismos son agentes oportunistas de la mucosa previamente dañada ${ }^{1}$. El organismo más frecuentemente detectado es Klebsiella ozaenae, pero también se ha encontrado Staphylococcus aureus, Proteus mirabilis, Escherichia coli, entre otras.

Los factores etiológicos asociados a la RA primaria se mencionan en la Tabla 1.

$R A$ secundaria. La RA secundaria se produce como consecuencia a una condición determinada que actúa como factor principal desencadenante. En la Tabla 2 se describen los principales factores asociados al desarrollo de esta entidad, destacando por su frecuencia la cirugía rinosinusal.

\section{Patología}

Los principales hallazgos histológicos de la RA son cambios en la membrana mucosa desde epitelio cilíndrico pseudoestratificado ciliado a epitelio cúbico o escamoso estratificado (con islas de metaplasia), atrofia de las glándulas serosas y mucinosas, pérdida de cilios, pérdida de células caliciformes e infiltrado celular inflamatorio crónico ${ }^{1,2,6}$. Estos cambios producen un clearance mucociliar defectuoso que conduce a estasis de secreciones, que posteriormente se secan para formar costras que secundariamente son colonizadas e infectadas por microorganismos patológicos ${ }^{1,2}$.

La RA de larga data puede producir deformidad de la nariz en silla de montar debido a la resorción de los huesos nasales y el cartílago septa2 ${ }^{2,6}$.

Hay dos tipos histológicos de RA: Tipo I (50\%$80 \%$ de los casos) que se caracteriza por endarteritis obliterante, periarteritis y fibrosis periarterial de las arteriolas terminales; y tipo II (20\%-50\% de los casos) que se caracteriza por vasodilatación capilar ${ }^{2}$.

Tabla 1. Factores etiológicos asociados a la RA primaria2,4

\begin{tabular}{|ll|}
\hline Factores & Descripción \\
\hline Genéticos & Antecedentes familiares en $15 \%-30 \%$ \\
Infecciosos & Kozaenae, $S$ aureus, $P$ mirabilis, $E$ coli \\
Desarrollo & Alteración neumatización del seno maxilar \\
Nutricionales & Déficit de fierro y vitamina A \\
Autonómicos & Vasoconstricción excesiva \\
Endocrinos & Déficit de estrógenos \\
Inmunológicos & Alergia tipo I e inmunidad celular y humoral alterada \\
\hline
\end{tabular}


Tabla 2. Principales etiologías de la RA secundaria ${ }^{1,2}$

\begin{tabular}{|ll|}
\hline Factores & Descripción \\
\hline Cirugía rinosinusal & $15 \%-71 \%$ presenta síntomas de RA posterior a una turbinectomía \\
Trauma maxilofacial & Traumas extensos con compromiso nasal importante \\
Procesos inflamatorios & Rinosinusitis aguda recurrente y crónica \\
Trastornos granulomatosos & Tuberculosos, lupus vulgaris, sífilis, lepra, rinoescleroma, VIH \\
Externos & Radiación, irritantes, tóxicos (cocaína) \\
Anatómicos & Desviación septal importante \\
\hline
\end{tabular}

\section{Características clínicas}

La RA primaria, por lo general, comienza en la pubertad. Es seis veces más común en las mujeres y suele ser bilateral ${ }^{2,4,7}$. La RA secundaria comienza después de que intervengan los factores patógenos mencionados anteriormente, pero la evolución en el tiempo y la relación causa-efecto se desconoce ${ }^{1}$.

Los síntomas en ambos tipos son básicamente los mismos, siendo principalmente la obstrucción nasal, secreción purulenta, costras nasales diarias, sequedad nasal y mal olor ${ }^{1,7}$. Otros síntomas comunes incluyen dolor y presión facial, epistaxis, anosmia, cacosmia, halitosis, trastornos del sueño, dolor de cabeza, malestar general y depresión ${ }^{1,2,4,8}$. Menos frecuentemente, los pacientes presentan sensación de sequedad en la garganta, sensación de cuerpo extraño y asfixia, lo cual se produce cuando las costras caen de la nasofaringe a la orofaringe $e^{2,4}$.

La causa de la congestión nasal paradójica en RA sigue siendo desconocida. Varias investigaciones sugieren que esto puede ser causado por una incapacidad para detectar flujo de aire en la nariz, lo cual resulta en la sensación de congestión nasal, probablemente debido a la atrofia de los receptores sensoriales de la mucosa nasal. Otra explicación para esto es que la resistencia nasal proporcionada por las estructuras intranasales puede ser necesaria para equilibrar la resistencia pulmonar durante la inspiración, y la falta extrema de resistencia nasal es percibida como obstrucción nasal paradójica'

Los signos más frecuentes son anatomía anormal de la pared lateral nasal (ausencia total 0 parcial de cornetes inferiores y ausencia de cornete medio), perforación del tabique nasal, mucosa nasal opaca, costras de color amarillo / verde / marrón, hedor y secreción mucopurulenta de los senos maxilares y etmoidales, la cual es observada en el meato medio. También se pueden observar las características clínicas de las secuelas ${ }^{1,2}$.

Las secuelas y complicaciones incluyen perforación del tabique nasal y deformidad de la nariz silla de montar, rinosinusitis secundaria, diseminación local y sistémica de la infección, faringitis y laringitis atrófica, dacriocistitis crónica y miasis nasal ${ }^{2}$.

\section{Diagnóstico}

El diagnóstico se realiza por un alto índice de sospecha clínica, descartando las causas secundarias de la RA y otras enfermedades granulomatosas. Dentro del estudio se incluye historia clínica, examen físico nasal, nasofibroscopía, pruebas de alergia, hemograma, VDRL, cultivos nasales y tomografía computarizada (TC) de la nariz y senos paranasales.

Otras investigaciones incluyen VHS, capacidad de fijación de hierro total y medición del nivel total de hierro sérico, proteínas séricas y niveles plasmáticos de vitaminas, batería autoinmune, la migración de leucocitos y las pruebas de rosetas espontáneas, punción sinusal, radiografía de tórax, punción del lóbulo de la oreja, rinomanometría, y biopsia nasal1,2,4,5.

Pace-Balzan y col$^{9}$ describieron los hallazgos característicos en la TC de la rinitis atrófica: 1) engrosamiento de la mucosa de los senos paranasales, 2) pérdida de definición del complejo osteomeatal secundario a la destrucción de la bulla etmoidal y el proceso uncinado, 3) hipoplasia del seno maxilar, 4) ampliación de las cavidades nasales con destrucción de la pared lateral nasal, y 5) destrucción ósea de los cornetes inferiores y medios. La disminución de la neumatización anteroposterior de los senos maxilares también se puede ver ${ }^{9}$.

\section{Tratamiento}

No existe tratamiento curativo para la $\mathrm{RA}^{4}$, por lo que, el objetivo del tratamiento es la reducción de los 
síntomas para mejorar la calidad de vida, eliminando las infecciones bacterianas secundarias, reducir la cantidad de costras, y disminuir el olor asociado ${ }^{8}$.

- Tratamiento médico: Consiste principalmente en la educación en cuanto a higiene nasal y el uso de antibióticos. La higiene nasal continua con irrigación de alta presión y de uso regular, sigue siendo el estándar de la terapia conservadora ${ }^{1}$. Las medidas humectantes deben ir acompañadas de la eliminación de las costras ${ }^{5}$. La terapia antibiótica sistémica debe ser guiada por los resultados de los cultivos nasales ${ }^{1}$. Los antibióticos de preferencia, teniendo en cuenta los principales patógenos, son los aminoglucósidos, rifampicina $600 \mathrm{mg}$ una vez al día durante 12 semanas, y ciprofloxacino 500 a 750 mg dos veces al día durante 8 a 12 semanas $^{1,2,5}$. En los casos de desnutrición y de deficiencias de hierro, zinc, proteínas y vitamina $A$, se recomiendan los suplementos ${ }^{2}$. Los vasoconstrictores y esteroides tópicos están contraindicados.

- Tratamiento quirúrgico: Los principios de la cirugía para la RA son la disminución del tamaño de las cavidades nasales, promover la regeneración de mucosa nasal normal, aumentar la lubricación de la mucosa nasal seca y mejorar la vascularización de las cavidades nasales². Existen varios procedimientos quirúrgicos para tratar la RA y cada cirugía intenta cerrar o estrechar la cavidad nasal y hacer el paso de aire más fisiológico ${ }^{8}$. Young describió el cierre bilateral de las fosas nasales, luego se describió la modificación del procedimiento de Young; ambos procedimientos han demostrado la desaparición de la formación de costras a los 6 meses después de la operación ${ }^{1}$, pero estas operaciones dificultan la respiración nasal y el olfato ${ }^{5}$. Los resultados a corto plazo de los últimos procedimientos propuestos, mediante la implantación artificial de diversos materiales para restaurar el volumen intranasal, han sido alentadores ${ }^{5}$, pero se necesita seguimiento a largo plazo para evaluar la reabsorción y la mejoría permanente ${ }^{1}$.

- Rinitis atrófica unilateral (RAU): La RAU se ha observado en pacientes con desviación importante del tabique nasal ${ }^{2,3}$. Tilley ${ }^{10}$ en 1912 reportó un caso en el que la paciente presentaba RAU derecha asociada a desviación del tabique nasal hacia el lado izquierdo. Bunnag y $\mathrm{col}^{7}$ observaron que en pacientes que presentan el tabique nasal desviado, las costras se encontraron sólo en el lado más ancho de la cavidad nasal.

\section{Etiología y patología}

La región anterior de las fosas nasales (principalmente el extremo anterior del cornete inferior) está expuesta a un alto flujo de aire y hay evidencia que indica que la estructura normal y la histología del epitelio nasal está influenciada por la exposición al flujo de aire normal. El flujo de aire a través de los conductos nasales es generalmente asimétrico y alternante debido al ciclo nasal y esto permite la recuperación del epitelio nasal expuesto al flujo de aire a alta velocidad ${ }^{11}$.

La desviación del tabique nasal a un lado de la nariz causaría cambios crónicos en el flujo de aire, con una reducción en el flujo de aire nasal ipsilateral y aumento del flujo de aire contralateral ${ }^{11}$. García y $\mathrm{col}^{12}$ sugirieron que la RAU asociada con desviación septal, se produce debido a que la cavidad en el lado cóncavo de tabique es demasiado amplio, por lo que el aire fluye en su mayoría a través de este lado, sometiéndolo a un gradiente permanente de agua y temperatura. Si el gradiente de agua es suficientemente grande y la mucosa está alterada, la capa mucosa podría secarse en las regiones de alto flujo de agua, conduciendo a la formación de costras y predisponiendo a la infección. Este flujo de aire continuo en el lado amplio provoca un aumento en la extensión del epitelio escamoso en la parte anterior de la nariz, que está expuesta al flujo de aire a alta velocidad, junto con una disminución de las células ciliadas y células caliciformes ${ }^{11}$; estos resultados son los mismos que se encuentran en los estudios histopatológicos de los pacientes con $\left.\mathrm{RA}^{1,2,4,6,8}\right)$.

En cuanto a los diagnósticos diferenciales es importante considerar la infección crónica rinosinusal unilateral, la supuración adenoidea en los adolescentes y cuerpos extraños o rinolitos².

\section{Tratamiento}

Garcia y $\mathrm{col}^{12}$ simularon el flujo de aire y los procesos de transporte que se producen en las cavidades nasales de cuatro pacientes sanos y de un paciente con RA (paciente con ausencia de cornetes), encontrándose una distribución anormal de los flujos de aire en este último, lo cual produce alteraciones en los flujos de agua en las paredes nasales. Estas 
simulaciones mostraron que en la fosa nasal atrófica no se produce un adecuado acondicionamiento del aire, debido a la alteración del transporte del agua, lo cual es consistente con la hipótesis de que la excesiva evaporación de la secreción nasal tiene un rol en la fisiopatología de esta enfermedad. Estos resultados sugieren que los principales objetivos de una cirugía deben ser restaurar la superficie original de la nariz, restaurar la distribución fisiológica del flujo de aire y crear cavidades simétricas.

Se ha reportado mejoría en los síntomas de la RAU después de la corrección quirúrgica de la desviación del tabique nasal; esto puede deberse a la división más uniforme de la tarea de humidificación entre las cavidades después de la cirugía. Esto sugiere que el ciclo nasal puede tener un papel importante en otorgar un período de descanso para la recuperación del epitelio nasal de los daños causados por el flujo de aire ${ }^{12}$. Es necesario considerar el riesgo de dejar al paciente con rinitis atrófica en ambos lados, en lugar de un $0^{10}$.

\section{CONCLUSIONES}

La rinitis atrófica es una enfermedad crónica y progresiva de la mucosa nasal que produce alteraciones significativas de la calidad de vida, principalmente por la fetidez, ocena, causada por la secreción purulenta y costras de las cavidades nasales. El tratamiento no es curativo, por lo que se han desarrollado distintas alternativas terapéuticas, principalmente conservadoras.

La rinitis atrófica unilateral se ha asociado con la desviación septal, lo que causa una alteración del ciclo nasal que produce las alteraciones patológicas características de la rinitis atrófica, por lo que su corrección quirúrgica se considera como una opción terapéutica.

El correcto diagnóstico etiológico de la rinitis atrófica permitirá entregar al paciente una mejor alternativa terapéutica, disminuyendo las infecciones bacterianas secundarias y reduciendo la formación de costras, lo cual entregará al paciente una mejor calidad de vida.

\section{BIBLIOGRAFÍA}

1. Moore EJ, Kern EB. Atrophic rhinitis: A review of 242 cases. Am J Rhinol 2001; 15: 355-61.

2. Dutt SN, Kameswaran M. The aetiology and management of atrophic rhinitis. J Laryngol Otol 2005; 119: 843-52.

3. Ly TH, Deshazo RD, Olivier J, Stringer SP, Daley W, Stodard CM. Diagnostic Criteria for Atrophic Rhinosinusitis. Am J Med 2009; 122(8): 747-53.

4. Mostafa AS. Atrophic rhinitis. Am J Otolaryngol 1996; 17(2), 81-6.

5. Hildenbrand T, Rainer KW, Brehmer D. Rhinitis sicca, dry nose and atrophic rhinitis: a review of the literature. Eur Arch Otorhinolaryngol 2010; 268(1): 17-26.

6. Çelik HH, Sargon MF, Özdemir MB, Ünal F, Önercl $M$. Scanning electron microscopic examination of the nasal mucosa epithelium in atrophic rhinitis. Anatomy 2008; 2: 34-8.

7. Bunnag $C$, Jareoncharsio $P$, Tansuriyawong $P$, Bhothisuman W, Chantarakul $N$. Characteristics of atrophic rhinitis in Thai patients at the Siriraj Hospital. Rhinology 1999; 37: 125-30.

8. Chand MS, Macarthur CJ. Primary atrophic rhinitis: A summary of four cases and review of the literature. Otolaryngol Head and Neck Surg 1997; 116: 554-8.

9. Pace-Balzan A, Shankar L, Hawke M. Computed tomographic findings in atrophic rhinitis. $J$ Otolaryngol 1991; 20: 428-32.

10. TILLEY H. Unilateral atrophic rhinitis in which the ostium of the right sphenoidal sinus is well seen. Proc R Soc Med 1912; 5(Laryngol Sect): 176.

11. BoYCE J, ECCLES R. Do chronic changes in nasal airflow have any physiological or pathological effect on the nose and paranasal sinuses? A systematic review. Clin Otolaryngol 2006; 31(1): 15-9.

12. García GJ, Ballie N, Martins DA, et al. Atrophic rhinitis: A CFD study of air conditioning in the nasal cavity. J Appl Physiol 2007; 103: 108292.

Dirección: Constanza J. Valdés

Avda. Salvador 364

E mail: cjvaldes@gmail.com 